\title{
La sécheresse de 1989
}

\author{
Yves Mérillon * et Pierre Chaperon **
}

La sécheresse de 1989, dont le début remonte en fait à la fin de 1988, aura été exceptionnelle tant par sa durée que par son intensité. A ce titre, elle est tout à fait comparable aux grandes sécheresses du dernier demi-siècle : 1949, 1976, 1985 et 1986 dans le sud-ouest.

Ses effets auront été durement ressentis par de nombreux secteurs économiques, au premier rang desquels l'agriculture. Le milieu naturel, lui aussi, a été particulièrement touché : eutrophisation, assèchement de nombreuses rivières, etc...

Il est indispensable d'établir le bilan d'un tel événement, afin d'en tirer tous les enseignements. De la même manière que la sécheresse de 1976 avait fait prendre conscience entre autres - de la nécessité de renforcer la sécurité de l'alimentation en eau potable, il est probable que celle de 1989 sera l'occasion de redéfinir l'ensemble de la politique de l'eau menée jusqu'à présent et d'en renforcer certains de ses aspects : c'est bien le principal élément positif de l'événement climatique exceptionnel que la France vient de connaître.

Nota : Un certain nombre de cartes et graphes illustrant cette note ont été élaborés par le bureau de l'hydraulique agricole et des grands ouvrages du ministère de l'agriculture et de la forêt, ainsi que par le bureau de recherches géologiques et minières.

Qu'ils soient remerciés de leur contribution.

\section{Pluviométrie et températures}

\section{I.I. Les précipitations}

a) Sur une année (novembre 1988-octobre 1989)

De novembre 1988 à octobre 1989, le total des précipitations sur la France continentale est de $560 \mathrm{~mm}$ (moyenne de 31 stations de la météorologie nationale réparties sur l'ensemble du territoire), ce qui représente $73 \%$ de la normale.

Cette valeur est la plus faible des trente deux dernières années (1957-1989) et sa récurrence peut être estimée à une année sur cent $(F=0,99)$. Il convient cependant d'apprécier cette récurrence avec prudence en raison de la taille relativement réduite de l'échantillon ( 32 valeurs). Un tel déficit, pour rare qu'il soit, n'est pas sans précédent.

En 1948-1949, le total novembre-octobre a été de $440 \mathrm{~mm}$. Pour les années antérieures, nous ne disposons pas des observations permettant d’évaluer la pluviométrie

(*) Secrétaire du groupe sécheresse de la Mission interministérielle de l'eau. Secrétariat d'Etat auprès du premier ministre chargé de l'environnement et de la prévention des risques technologiques et naturels majeurs. Service de l'eau.

$\left({ }^{* *}\right)$ Directeur de recherches, ORSTOM, Département des eaux continentales.

\section{Drought in France in 1989}

The 1989 french drought will remain in the memories as one of the longest and most severe of this century. It affected many economical activities, and at first agriculture and power plants. Water resources also suffered from high pollution problems and from the dry up of several streams. Yet, drinking water distribution could be ensured in most cases, with no major difficulties. The lessons drawn from such an event will have a strong influence on all the future water policies. 


\begin{tabular}{|c|c|c|c|}
\hline & & & $\begin{array}{c}\text { Rapport } \\
\text { à la } \\
\text { normale }\end{array}$ \\
\hline Nord & $559 \mathrm{~mm}$ & valeur observée 1 année sur & $85 \%$ \\
\hline Nord-Est & $663 \mathrm{~mm}$ & valeur observée 1 année sur & $86 \%$ \\
\hline Ouest & $579 \mathrm{~mm}$ & valeur observée 1 année sur 20 & $74 \%$ \\
\hline Centre-Est & $546 \mathrm{~mm}$ & valeur observée 1 année sur 100 & $69 \%$ \\
\hline Sud-Ouest & $631 \mathrm{~mm}$ & valeur observée 1 année sur 100 & $73 \%$ \\
\hline Sud-Est & $384 \mathrm{~mm}$ & valeur observée 1 année sur 50 & $56 \%$ \\
\hline Corse & $434 \mathrm{~mm}$ & valeur observée 1 année sur 20 & $67 \%$ \\
\hline
\end{tabular}

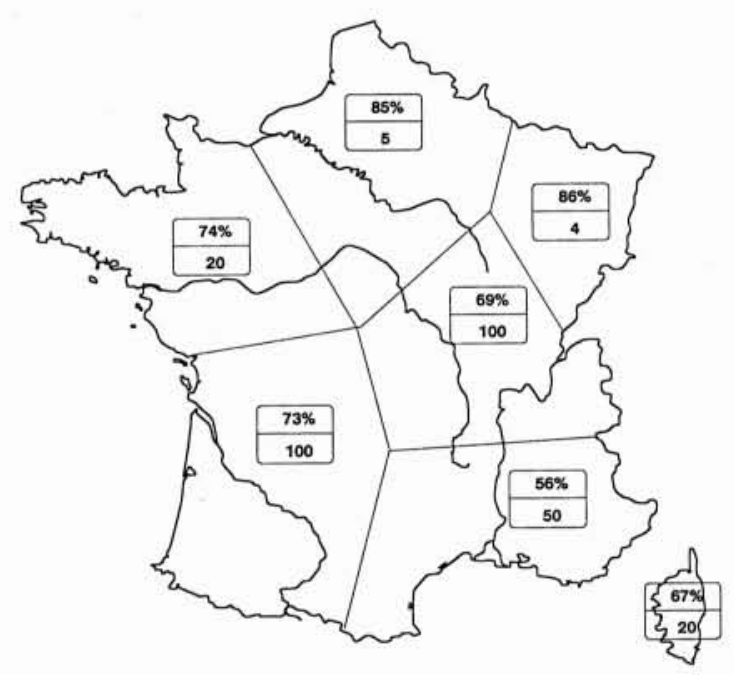

85\% rapport à la normale

5 période de retour (une année sur cinq)

1. Pluviométrie de l'année novembre 1988-octobre 1989.
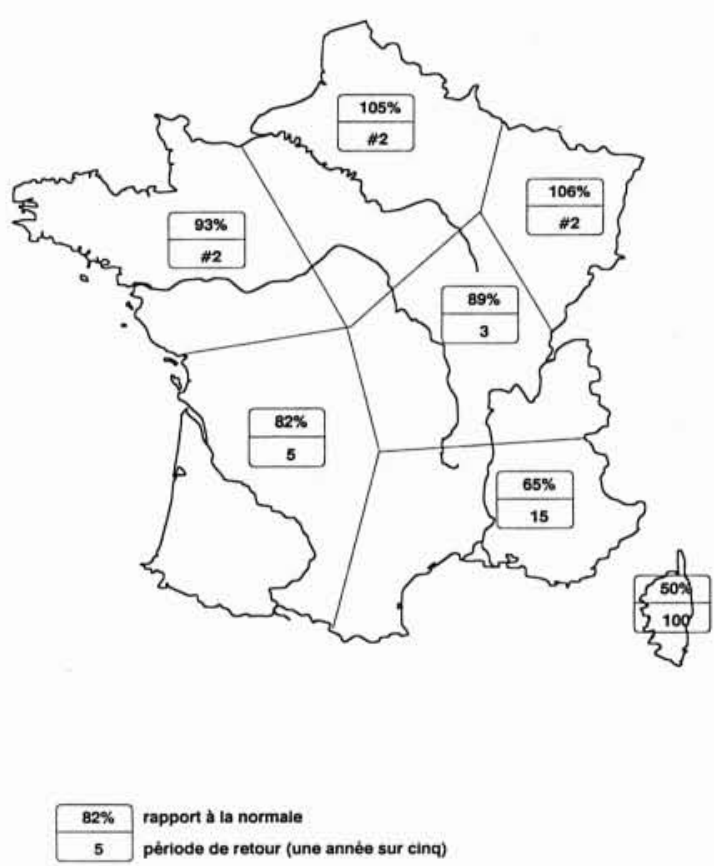

nota : \#2 voisine de la normale

2. Pluviométrie du semestre novembre 1988-avril 1989.

\begin{tabular}{|c|c|c|c|c|}
\hline Région & $\begin{array}{c}\text { Total } \\
\text { novembre-avril }\end{array}$ & $\begin{array}{c}\text { Ecart à la } \\
\text { normale }\end{array}$ & $\%$ & Récurrence \\
\hline $\begin{array}{l}\text { Nord } \\
\text { Nord-Est } \\
\text { Ouest } \\
\text { Centre-Est } \\
\text { Sud-Ouest } \\
\text { Sud-Est } \\
\text { Corse }\end{array}$ & $\begin{array}{l}333 \mathrm{~mm} \\
372 \mathrm{~mm} \\
414 \mathrm{~mm} \\
309 \mathrm{~mm} \\
384 \mathrm{~mm} \\
243 \mathrm{~mm} \\
208 \mathrm{~mm}\end{array}$ & $\begin{array}{l}+15 \mathrm{~mm} \\
+\quad 20 \mathrm{~mm} \\
-30 \mathrm{~mm} \\
-\quad 37 \mathrm{~mm} \\
-\quad 83 \mathrm{~mm} \\
-133 \mathrm{~mm} \\
-204 \mathrm{~mm}\end{array}$ & $\begin{array}{r}105 \\
106 \\
93 \\
89 \\
82 \\
65 \\
50\end{array}$ & $\begin{array}{l}\text { proche de la normale } \\
\text { proche de la normale } \\
\text { proche de la normale } \\
1 \text { année sur } 3 \\
1 \text { année sur } 5 \\
1 \text { année sur } 15 \\
1 \text { année sur } 100 \text { (?) }\end{array}$ \\
\hline
\end{tabular}




\begin{tabular}{|l|c|c|c|c|}
\hline Région & $\begin{array}{c}\text { Total } \\
\text { mai-octobre }\end{array}$ & $\begin{array}{c}\text { Ecart } \\
\text { la normale }\end{array}$ & $\%$ & Récurrence \\
\hline Nord & $226 \mathrm{~mm}$ & $-115 \mathrm{~mm}$ & 66 & 1 année sur 10 \\
Nord-Est & $291 \mathrm{~mm}$ & $-126 \mathrm{~mm}$ & 70 & 1 année sur 10 \\
Ouest & $165 \mathrm{~mm}$ & $-177 \mathrm{~mm}$ & 48 & 1 année sur 100 \\
Centre-Est & $237 \mathrm{~mm}$ & $-209 \mathrm{~mm}$ & 53 & 1 année sur 50 \\
Sud-Ouest & $247 \mathrm{~mm}$ & $-156 \mathrm{~mm}$ & 61 & 1 année sur 20 \\
Sud-Est & $141 \mathrm{~mm}$ & $-167 \mathrm{~mm}$ & 46 & 1 année sur 20 \\
Corse & $226 \mathrm{~mm}$ & $-5 \mathrm{~mm}$ & 98 & proche de la normale \\
\hline
\end{tabular}

moyenne annuelle, mais quelques indications chiffrées ou appréciations permettent de caractériser des sécheresses notables tout au long des siècles passés.

Le déficit de 1989 est donc un phénomène climatologique rare mais nullement exceptionnel. Il a principalement affecté la moitié sud-ouest de la France (au sud d'une ligne Caen-Nice) mais n'a pas épargné l'est du pays.

Par région, les valeurs suivantes ont été observées sur douze mois - novembre 1988 à octobre 1989 (fig. I, tabl. I).

\section{b) Par semestre}

Le premier semestre (novembre-avril) est resté voisin ou même supérieur à la normale sur la moitié nord-est de la France. La sécheresse (déficit de tous les mois) au cours du second semestre (mai-octobre) s'est étendue à la plus grande partie du territoire français.

\section{Le premier semestre (novembre 1988-avril 1989)}

Les quatre premiers mois sont généralement déficitaires. Une certaine stabilisation est observée en février et mars. Avril est partout très excédentaire ( 2 à 3 fois la normale) :

Le bilan du semestre est le suivant (fig. 2, tabl. II).

\section{Le second semestre (mai 1989-octobre 1989)}

A l'exception de la Corse, où la pluviométrie se redresse en septembre et octobre, tous les mois de mai à octobre sont déficitaires : le déficit cumulé reste voisin ou inférieur à ceux de 49 et 76 jusqu'en août mais rejoint (ou dépasse nettement comme dans l'ouest) les déficits de 49 et 76 (86 dans le sud-ouest) à partir du mois de septembre à la suite d'une prolongation de la sécheresse en début d'automne. (Cf. fig. 3 les graphiques des écarts cumulés de l'été) :

Mai est très déficitaire (et chaud) : moins de $40 \%$ de la normale sur près de la moitié en France.

Le déficit de juin est marqué (inférieur à $40 \%$ de la normale) dans la vallée du Rhône, le sud, d'Agen à Nice, et dans le Finistère.

En juillet, déficit (moins de $40 \%$ ) accusé en Bretagne, Picardie et dans l'ensemble du sud-est.

En août, déficit marqué dans les Pays de la Loire, la Bretagne, la Normandie et à l'est du Rhône.

En septembre, les régions les plus touchées sont le grand ouest, de la Bretagne au Limousin ainsi que l'est du Rhône.

En octobre, la moitié sud de la France reçoit moins de la moitié de la normale ainsi que le nord de la Bretagne.

Le bilan du semestre est le suivant (fig. 4, tabl. III).

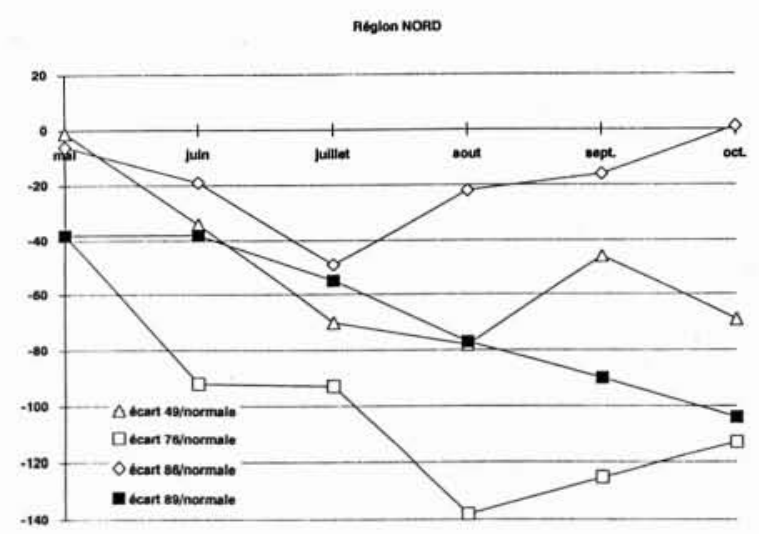

3. Pluviométrie : écarts cumulés au cours des étés 1949, 1976, 1986 et 1989.
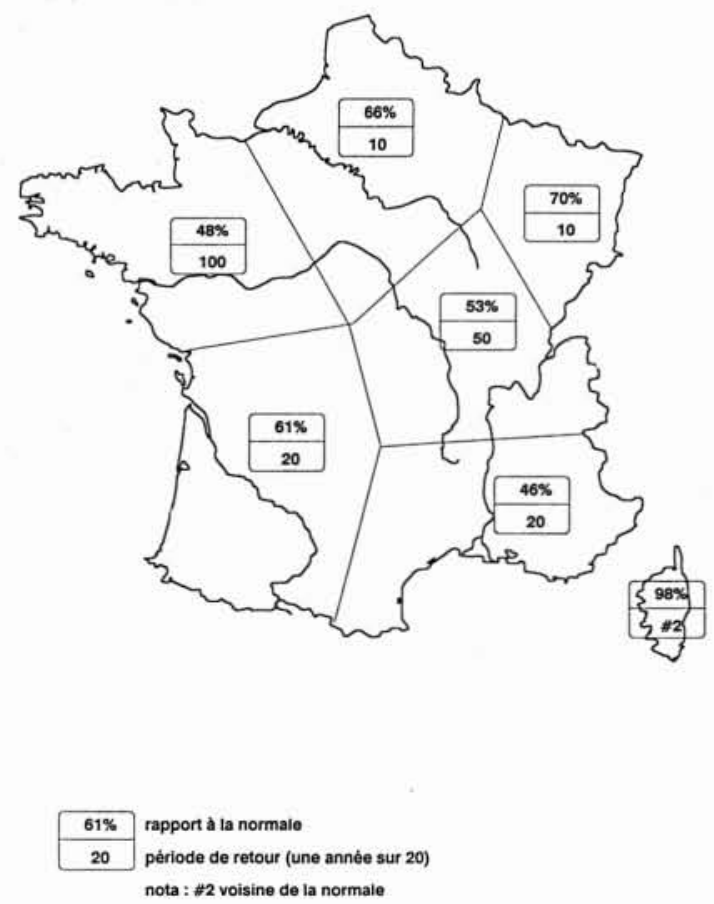

4. Pluviométrie du semestre mai 1989-octobre 1989 


\section{c) La fin de l'année 1989}

Les deux derniers mois de 1989 auront connu deux périodes très pluvieuses: les premiers jours de novembre et la deuxième décade de décembre.

Mais hormis ces deux épisodes, les pluies ont été soit totalement absentes soit très modérées.

Au total le mois de novembre s'est conclu par une pluviométrie déficitaire sur tout le territoire exception faite du centre est.

En décembre, les pluies tombées après le $10 \mathrm{du}$ mois ont principalement arrosé la moitié-nord de la France, la moitié-sud connaissant un mois supplémentaire de pluviométrie déficitaire.

\subsection{Les températures}

La période de 13 mois "septembre 1988-octobre 1989 " a été la plus chaude que la France ait connue au cours des trente dernières années. La moyenne des températures pour l'ensemble du pays a été de $12,7^{\circ} \mathrm{C}$. Elle bat donc celle des périodes correspondantes de 1960-1961 (12,5 $\left.{ }^{\circ} \mathrm{C}\right)$, de 1958-1959 $\left(12,4^{\circ} \mathrm{C}\right)$ et encore plus de 1975-1976 $\left(11,7^{\circ} \mathrm{C}\right)$.

Novembre et décembre 1989 auront été plus contrastés, alternant des périodes de douceur et des périodes de froid relativement marquées.

Ces températures élevées ont évidemment beaucoup contribué aux fortes consommations d'eau observées au cours de l'année.

\section{Hydrologie}

\subsection{Les indicateurs hydrologiques}

On examinera successivement l'évolution de la situation concernant :

- la teneur en humidité des sols ;

- les précipitations efficaces ;

- l'état des stocks naturels (manteau neigeux, nappes) et artificiels (barrages, réservoirs) ;

- les débits des cours d'eau.

\subsection{La teneur en humidité des sols}

Elle est caractérisée par le rapport de la réserve résiduelle (en fin de mois) à la réserve utile du sol $(R / R U)$.

La réserve utile est évaluée à partir des caractéristiques pédologiques du sol (sur 1 mètre), la réserve résiduelle étant calculée à partir du bilan des précipitations et de l'évapotranspiration potentielle.

Les valeurs obtenues, sans grande signification dans l'absolu, sont comparées aux valeurs observées sur une période de 30 années et permettent de comparer la situation des sols aux normales ainsi que de suivre l'évolution du taux d'humidité. Cet indicateur réagit rapidement aux flux (précipitations et évapotranspirations).
En 1989, dès le mois de janvier, le déficit de précipitations du début de l'hiver s'est reflété dans les valeurs anormalement faibles du rapport $R / R U$ : plus de la moitié de la France étant en dessous de $90 \%$ de la normale avec des valeurs records inférieures à $50 \%$. Cette situation, en janvier, n'est observée globalement qu'une année sur dix. Les zones déficitaires sont fortement réduites, fin février, en raison des fortes précipitations de la fin du mois. Le déficit s'étend ensuite en mars (moins de $75 \%$ sur la moitié-sud de la France) en raison d'une évapotranspiration supérieure à la normale (excédent thermique). Les fortes précipitations d'avril sont responsables d'un taux d'humidité partout très supérieur à la normale du mois. Mais de mai à octobre, le déficit pluviométrique cumulé et les températures supérieures à la normale entretiennent sur toute la période et la majeure partie du territoire, un déficit constant des taux d'humidité et obligent les agriculteurs à faire appel à des apports extérieurs en eau d'irrigation.

C'est ainsi, qu'en août par exemple, le rapport $R / R U$ est très faible sur la quasi-totalité du pays (inférieur à $40 \%$ ). Au sud d'une ligne Saint-Malo-Belfort ainsi que dans le nord de l'Alsace, les réserves sont nulles, ce qui correspond à une période de retour supérieure à 10 ans.

A la fin du mois de septembre, la sécheresse des sols affecte la plus grande partie du pays et compromet gravement la production fourragère, sans espoir d'obtenir en automne des repousses importantes. La mise en place des cultures d'automne est aussi très difficile.

\subsection{Les précipitations efficaces}

Elles correspondent au reliquat des précipitations totales, une fois satisfaits plus ou moins complètement, les besoins de l'évapotranspiration et de reconstitution du stock d'humidité des sols.

De l'importance des précipitations efficaces dépendent la recharge des nappes et les écoulements des cours d'eau.

La carte ci-après (fig. 5, source: B.R.G.M.) fournit la répartition des pluies efficaces de l'année hydrologique 1988-1989. Les régions situées au nord-est sont légèrement excédentaires par rapport à la normale alors que partout ailleurs les pluies efficaces sont très inférieures à ces moyennes ; elles peuvent être nulles, ce qui pour certaines régions du centre-ouest est exceptionnel : si ce phénomène survient une année sur deux à Montpellier ou Perpignan, il est beaucoup plus rare à Rennes (une année sur dix) ou à Bourges (une année sur vingt)

\subsection{Les stocks}

a) La constitution du manteau neigeux dépend en premier lieu des précipitations, puis des températures. Très faible jusqu'en février (absence presque totale de neige en zones de moyenne altitude), l'épaisseur du manteau neigeux a profité des précipitations de fin février-début mars, et surtout d'avril, mais la température relativement douce de la saison a nui à la conservation de ce stock. 


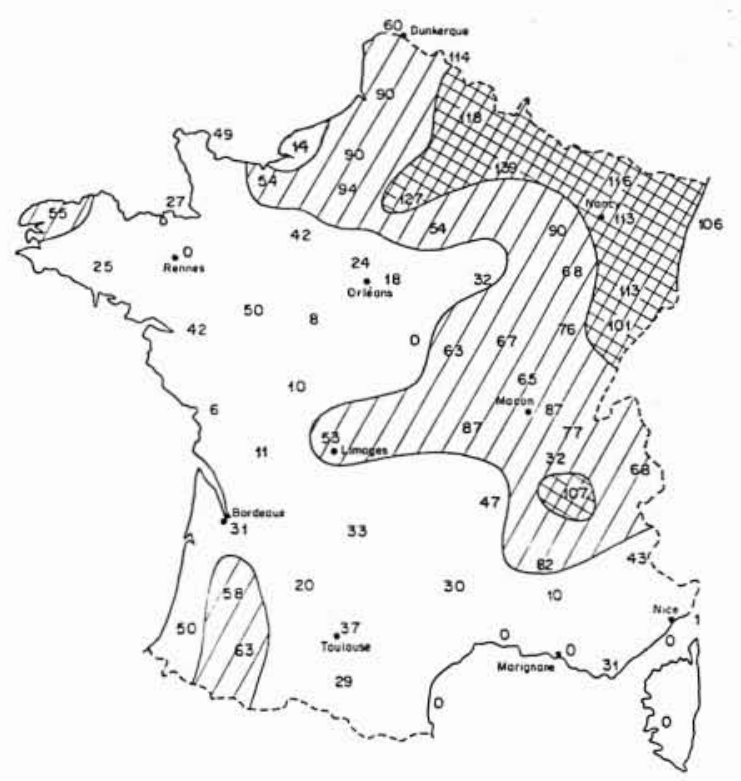

60 Voleut en pout sert de la pluie efficece de l'annite por ropeort

XX Plive etficoce superieure à lo mayenne

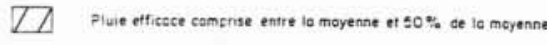

$\square$ Piue efticoes infentiture ó $50 \%$ ce la moyenne

5. Pluies efficaces de l'année 1988-1989: comparaison avec la moyenne de référence (Source: BRGM).

6. Niveaux d'eaux et débits de sources relevés entre 1975 et 1989.

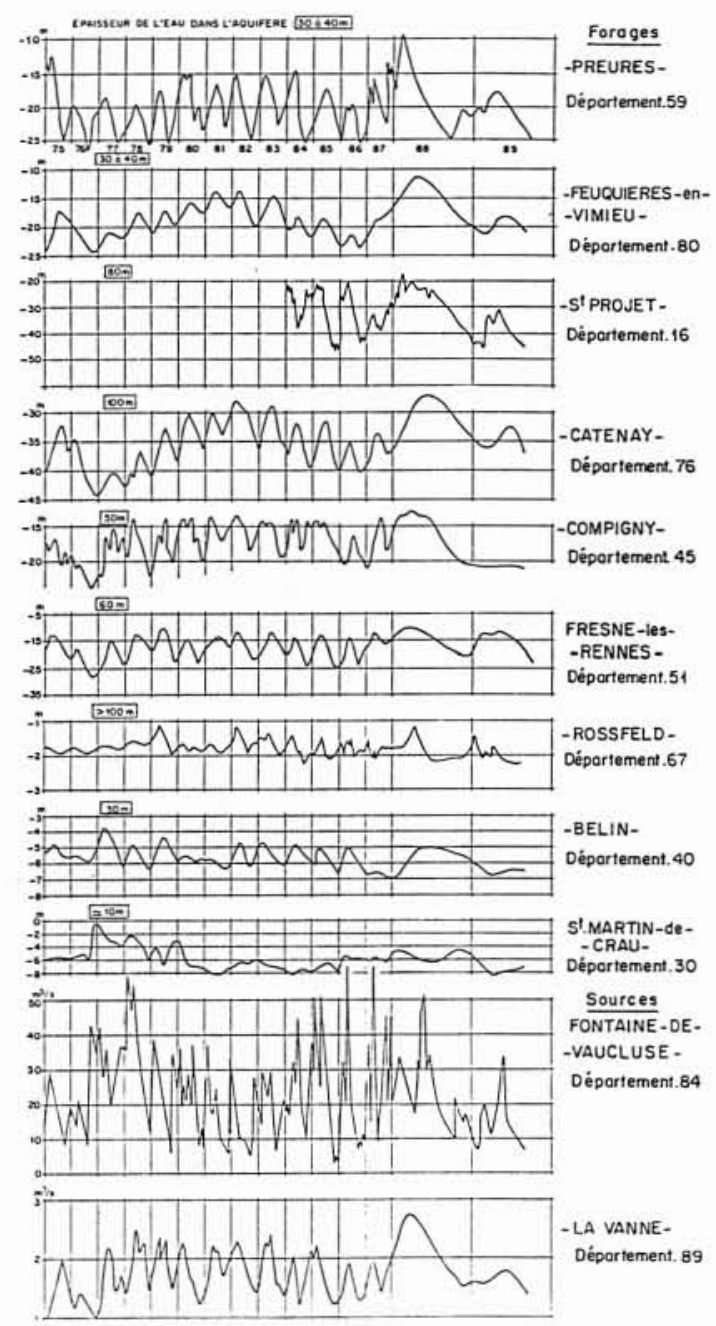

Fin avril, dans toutes les régions montagneuses, les stocks neigeux sont partout déficitaires, plus ou moins sévèrement, et ne connaîtront plus aucun apport (40 à $70 \%$ de la normale dans les Alpes du sud, $60 \%$ de la normale dans les Pyrénées). Cette situation se reflètera dès le printemps et durant l'été sur l'hydraulicité des cours d'eau.

b) Les répercussions de la sécheresse sur les nappes d'eau souterraines dépendent de leur nature et de leur situation.

Les grands aquifères à piézométrie continue situés dans les formations fissurées (craies, calcaires) des grands bassins sédimentaires (nappe du calcaire carbonifère de l'Artois, du calcaire de Champigny, etc...) ou dans les formations poreuses (sables, alluvions) des grands bassins alluviaux (plaine d'Alsace, nappe de la Craie, etc.) sont, en raison de leur régulation interannuelle, peu sensibles aux précipitations estivales. Les flux ne représentent qu'une fraction relativement faible des stocks (épaisseur des nappes généralement de plusieurs dizaines de mètres). En début 1989, ces nappes étaient généralement en position haute en raison de l'hydraulicité excédentaire des dix dernières années et elles n'ont que faiblement répercuté le déficit de 1989. A l'exception du sud de la France, où la sécheresse de 1976 avait été moins prononcée, elles sont restées géné- ralement au-dessus des niveaux de 1976, année précédée d'une décennie d'hydraulicité déficitaire. La figure 6 ciaprès (source : B.R.G.M.) présente une sélection de chroniques de niveaux d'eau relevés sur certaines grandes nappes.

Il n'en va pas de même pour les nappes situées sur le socle ancien (Massif Armoricain, Massif Central, Vosges Cristallines) et dans les zones montagneuses. Ces nappes localisées, à piézométrie discontinue, sont très sensibles aux précipitations récentes. Elles ont donc répercuté le déficit de 1989 et sont très souvent descendues au-dessous des niveaux de 1976.

c) La situation des réservoirs artificiels a été très variable.

Les réservoirs saisonniers d'Electricité de France ont connu en moyenne une situation déficitaire. Le taux moyen de remplissage des lacs artificiels est resté voisin du minimum constaté (période 1978-1988) au cours du dernier trimestre de 1988. La grève des personnels du secteur thermique a également eu pour conséquence une forte sollicitation des réservoirs.

En raison de la faible pluviométrie de l'hiver, le taux de remplissage s'est ensuite écarté très sensiblement des valeurs minimales antérieures. 


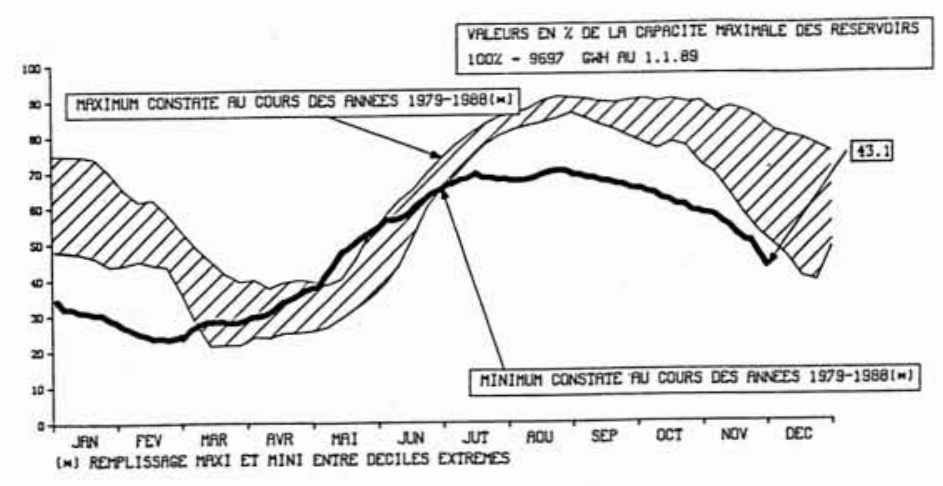

\begin{tabular}{|c|c|c|c|c|c|}
\hline LAC & CENTRRLE DE TETE & $z$ & LAC & CENTRALE DE TETE & $z$ \\
\hline $\begin{array}{l}\text { VOUGLANS } \\
\text { ROSELENO } \\
\text { TIGNES } \\
\text { MONT-CENIS } \\
\text { BISSORTE } \\
\text { GRAND' HAISON } \\
\text { SAUTET } \\
\text { CRSTIULN } \\
\text { MONTEYNPRO } \\
\text { SERRE-PONCON } \\
\text { SAINTE-CRDIX }\end{array}$ & $\begin{array}{l}\text { VOUGLPNS } \\
\text { LA BRTHIE } \\
\text { BREVIERES-HALEOVERT } \\
\text { VILPROOIN-COMBE D'A } \\
\text { BISSORTE } \\
\text { GRRNO' HAISON } \\
\text { SAUTET-CORDEAC } \\
\text { CASTIULN } \\
\text { MONTEXNPRO } \\
\text { SERRE-PONCON } \\
\text { SAINTE-CROIX }\end{array}$ & $\begin{array}{l}21.95 \\
28.23 \\
44.58 \\
46.79 \\
17.24 \\
28.09 \\
14.56 \\
56.20 \\
31.98 \\
51.80 \\
53.21\end{array}$ & $\begin{array}{l}\text { CHASSEZAC } \\
\text { MONTPEZRT } \\
\text { BORT LES ORLUES } \\
\text { L'AIGLE } \\
\text { GRRNOVRL } \\
\text { SARRPNS } \\
\text { HONTFHUT LFOUZAS } \\
\text { PARELOUP } \\
\text { CAP DE LONS } \\
\text { LANOUX } \\
\text { NAGUILES }\end{array}$ & 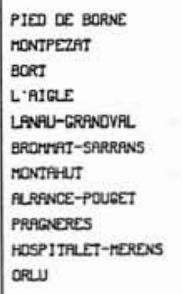 & $\begin{array}{l}60.91 \\
70.28 \\
24.50 \\
80.29 \\
27.20 \\
59.06 \\
81.90 \\
11.12 \\
55.88 \\
14.25 \\
42.16\end{array}$ \\
\hline
\end{tabular}

7. Niveaux des réservoirs E.D.F. au cours de l'année 1988-1989 (Source: Ministère de l'industrie).

Au cours du deuxième trimestre 1989 la courbe de remplissage s'est fortement redressée en raison d'une part de la pluviométrie excédentaire d'avril mais également d'une très faible sollicitation des réserves par E.D.F. Puis de juillet à octobre, les niveaux moyens sont restés à nouveau très inférieurs aux situations minimales antérieures (65 à $55 \%$ de juillet à octobre contre 75 à $80 \%$ ) (fig. 7 : source : ministère de l'industrie).

Les grands ouvrages à buts multiples (écrêtement des crues et soutien d'étiage) des bassins de la Seine et de la Loire ont généralement pu atteindre leurs objectifs de remplissage et participer efficacement pendant l'été au soutien des étiages naturels déficitaires.

Les retenues à usage hydro-agricole (soutiens d'étiages et irrigation) sont en général dimensionnées pour assurer un remplissage garanti quatre années sur cinq.

Fin février, les objectifs de remplissage étaient très loin d'être atteints et la situation préoccupante. Les précipitations de mars et d'avril ont permis de conforter la situation d'une grande partie des retenues mais, dans le sud-ouest et le Languedoc-Roussillon, la situation en début d'été était restée très critique.

Dans ces régions, fin juin 1989, les taux de remplissage étaient les suivants :

$\begin{array}{lll}\text { La Bancalie } & \text { (Tarn-Aveyron) } & 63 \% \\ \text { Mondelys } & \text { (Garonne-Pyrénées) } & 44 \% \\ \text { Lavaud } & \text { (Charentes) } & 45 \% \\ \text { Montbel } & \text { (Garonne-Pyrénées) } & 54 \% \\ \text { Montagne Noire } & \text { (Tarn-Aveyron) } & 54 \%\end{array}$

La retenue des Cammazes, dans le Lauragais, remplie à $60 \%$ seulement n'a pas permis de remplir ses objectifs d'irrigation et d'alimentation du canal du midi qui ont dû être interrompues.

\subsection{Les débits des cours d'eau}

Pendant la période estivale, les débits des cours d'eau sont essentiellement alimentés par la vidange des magasins naturels (nappes de soutien d'étiage). Ces nappes, lorsque l'été est moyennement pluvieux peuvent être partiellement rechargées et il est possible d'observer à l'occasion d'épisodes pluvieux intenses, des crues épisodiques se greffant sur le débit de base. En 1989, l'absence quasi totale de précipitations efficaces ainsi que la situation déficitaire en fin de printemps des stocks naturels (nappes et manteau neigeux) ont été la cause d'une baisse rapide des débits qui, dans la grande majorité du territoire, ont atteint ou dépassé les records déficitaires antérieurs.

Le bureau de l'hydraulique agricole et des grands ouvrages du ministère de l'agriculture et de la forêt, gestionnaire de la banque nationale HYDRO, a suivi l'évolution de la situation en temps quasi réel (compte tenu des délais de réunion des informations).

On trouvera ci-après, une série de cartes de situation au cours de l'été (fig. 8).

Au $1^{\text {er }}$ juin, les étiages sont accusés sur la plupart des cours d'eau des régions suivantes $(50 \%$ des cours d'eau avaient des débits d'étiages inférieurs à ceux constatés quatre années sur cinq - étiages quinquennaux): Est de la Bretagne, une partie de la Basse Normandie, grands affluents rive gauche de la Loire moyenne, Poitou et Charentes, Pyrénées, littoral méditerranéen, Corse. Les étiages sont exceptionnels (débits les plus bas observés à cette période pour plus de $50 \%$ des cours d'eau) en Provence, à l'est du Rhône, et en Corse du sud.

Au 15 juillet les $3 / 4$ de la France au sud d'une ligne Nantes-Belfort ainsi que la Bretagne et le Cotentin 
connaissent des étiages prononcés (quinquennaux pour la plupart des rivières). Ces étiages sont exceptionnels (records égalés ou battus) dans les Pyrénées, la Montagne Noire et au sud de la Loire.

Au $1^{\text {er }}$ septembre, la zone d'étiages prononcés s'étend au sud d'une ligne Caen-Belfort ainsi que dans le nord de l'Alsace et de la Lorraine et l'est du Bassin Parisien. Des étiages exceptionnels sont observés sur le littoral de la
Haute-Normandie, en Bretagne centrale, Vendée et Charentes, en Midi-Pyrénées et dans la Drôme.

Au 15 octobre, la plus grande partie de la France connaît des étiages sévères. Les zones à étiages exceptionnels se multiplient : Bretagne, Basse-Normandie, sud de la Loire, Limousin, Cévennes, Drôme, Haute-Marne, etc... Par contre la situation est redevenue normale ou excédentaire en Corse.

EVOLUTION DE L'ECOULEMENT AU COURS DE L'ETE 1989 (FREQUENCES)

COMPARAISON AVEC LES ECOULEMENTS DU PASSE AUX MEMES PERIODES OU LES ECOULEMENTS SONT-ILS LES PLUS EXCEPTIONNELS ?

SITUATION AU 1er JUIN

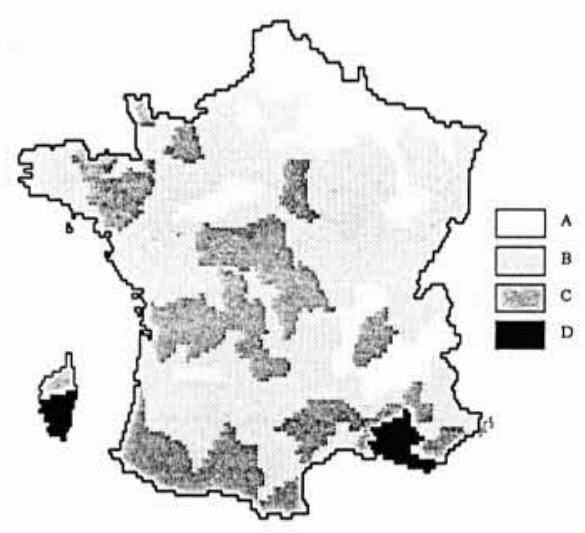

SITUATION AU 1er SEPTEMBRE

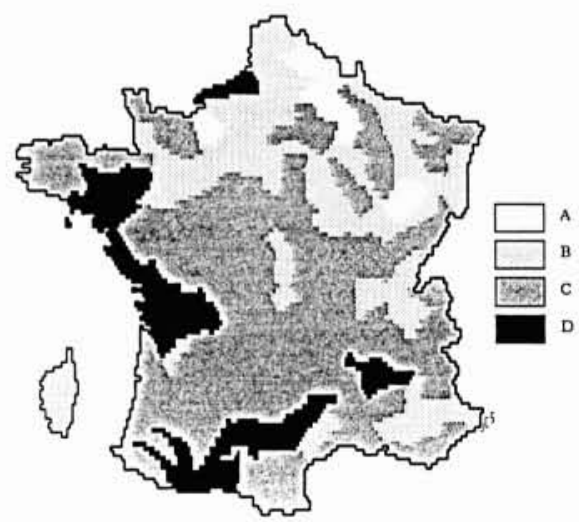

SITUATION AU 15 JUILLET

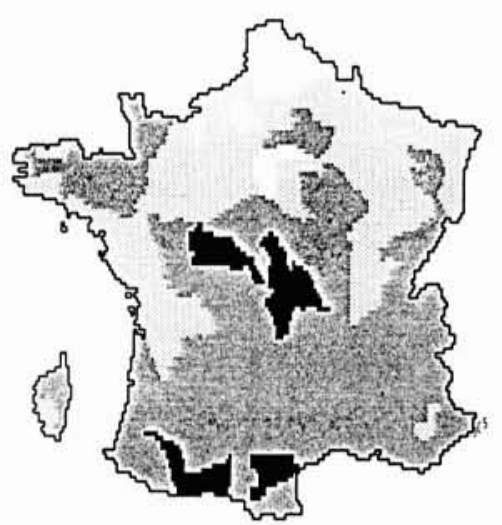

SITUATION AU 15 OCTOBRE

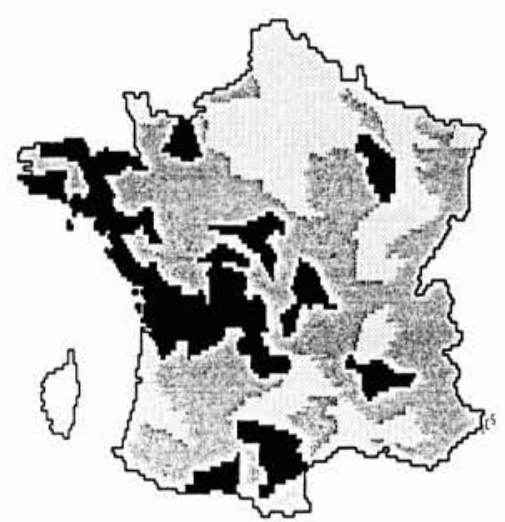

LEGENDE

DEBITS SUPERIEURS AUX ETIAGES HABITUEIS D $50 \%$ des cours d'eau cannaisent des déhits snpèrieurs aux étiağes habituels en cette QUELQUES ETIAGES I me minorité de cours d'eau connaisent des étiages quinquennaux)

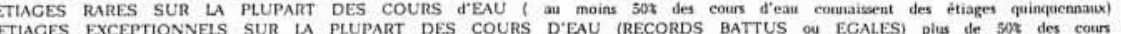

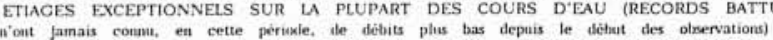

8. Débits des cours d'eau (fréquence) au cours de l'été 1989 (Source: Ministère de l'agriculture et de la forêt). 


\section{Le suivi et la gestion de la sécheresse par les pouvoirs publics. Les mesures prises au cours de l'été}

\subsection{Le suivi et la gestion de la sécheresse par les pouvoirs publics}

La Mission interministérielle de l'eau (M.I.E.) s'est saisie de cette question dès le mois de février 1989 : la sécheresse de l'hiver 1988/1989 laissait en effet présager dès cette époque des difficultés pour le printemps et l'été en cas de pluviométrie inférieure à la normale pendant le premier semestre.

Un suivi bimensuel a été effectué dans tous les bassins hydrographiques ainsi qu'à l'échelon national. La M.I.E. décidait au mois d'avril de créer un groupe de travail spécialisé dont la conduite et le secrétariat serait assuré par le Secrétariat d'Etat auprès du Premier ministre chargé de l'environnement et de la prévention des risques technologiques et naturels majeurs. Ce groupe de travail s'est réuni pendant toute la période de crise, environ toutes les deux ou trois semaines. A chaque réunion un bulletin hydrologique a été rédigé et diffusé (1 000 exemplaires environ) à la presse, aux services territoriaux de l'administration et aux distributeurs d'eau.

Devant la persistance de la sécheresse, ce groupe s'est adjoint au mois de juillet les représentants des distributeurs d'eau, de l'Association des maires de France et de la Fédération nationale des collectivités concédantes et régies.

Outre le rôle d'information déjà signalé, ce groupe a édité des directives et des notes circulaires pour aider les préfets à gérer la situation dans leur département et impulser certaines actions spécifiques :

- lettre circulaire du 8 juin 1989 rappelant les textes sur lesquels appuyer l'action de l'Etat et demandant, là où la situation l'exigeait, la création de groupes de travail " sécheresse " chargés d'établir la liste des mesures préventives et curatives susceptibles d'être mises en œuvre, d'assurer le suivi de l'évolution des ressources en eau dans la région considérée et de proposer au préfet les décisions à prendre ;

- lettre du secrétaire d'Etat auprès du Premier ministre chargé de l'environnement et de la prévention des risques technologiques et naturels majeurs aux préfets leur demandant le 23 août 1989 de redoubler la vigilance des services chargés de la police des eaux et de la surveillance des installations classées, en raison des graves conséquences d'une éventuelle pollution accidentelle au moment des plus basses eaux.

Au niveau local, des "cellules de crise " se sont réunies régulièrement dans de nombreux départements, régions ou même bassins (bassin Adour-Garonne). Ce sont ces cellules qui ont préparé les mesures prises par les autorités administratives (préfet, maire) et assuré la remontée de l'information vers la mission interministérielle de l'eau.

En complément de cette action administrative classique, le secrétariat d'Etat auprès du Premier ministre chargé de l'environnement et de la prévention des risques technolo- giques et naturels majeurs a mis au point, à la demande du cabinet du secrétaire d'Etat, une campagne de communication destinée à faire prendre conscience aux français de la nécessité d'économiser l'eau et de la solidarité naturelle entre les usagers. Cette campagne de communication, bâtie autour d'un animal symbolisant les économies d'eau (le zébu), s'est développée aux mois de juillet et août selon deux axes:

- spots radios : trois vagues sur France-Inter, Sud-Radio, Radio Monte-Carlo ;

- affiches, tracts, autocollants dans les départements les plus touchés.

Les messages, lus ou présentés par le zébu, donnaient des conseils très pratiques d'économie de l'eau. Cette campagne, financée aux trois quarts par les agences de bassin a coûté $4 \mathrm{MF}$.

\subsection{Les mesures prises pour économiser l'eau et accroître les ressources}

\section{a) Les mesures d'économie d'eau}

Dès lors qu'il est devenu évident que les réserves constituées à la sortie de l'hiver seraient insuffisantes pour assurer la couverture complète des besoins, les premières mesures d'économie ont été prises.

Les irrigants de la basse Durance ont, dès le mois de février, réduit leur consommation habituelle. L'économie réalisée pendant le premier semestre est estimée à $100 \mathrm{Mm}^{3}$.

Dans le sud-ouest, malgré le répit apporté par le mois d'avril très pluvieux, les premières mesures de restriction autoritaires ont dû être prises au mois de juin dans la région du Lauragais, qui fut le véritable épicentre de la sécheresse dans le sud-ouest. La décision fut prise très tôt de réserver à l'alimentation en eau potable l'eau emmagasinée par le réservoir des Cammazes.

Ce type de restrictions devait se généraliser à des degrés divers pendant tout l'été, les économies d'eau domestique demandées à la population étant généralement complétées par des limitations de l'irrigation : interdiction d'arroser quelques heures par jour, création de tours d'eau. Ces mesures sont en général devenues de plus en plus sévères au cours de l'été, pouvant aller jusqu'à l'interdiction totale d'utiliser certaines ressources.

A la fin du mois d'août, le nombre de départements ayant pris ce type de mesures dépassait la quarantaine, la quasi-totalité des départements situés au sud-ouest d'une ligne Caen-Marseille étant concernée. La figure 9 présente la situation à la fin de l'été.

Dans le sud-est, les mesures de restrictions autoritaires ont pu être, dans l'ensemble, évitées ou réduites grâce aux infrastructures des bassins de la Durance et du Verdon (réserves de Serre-Ponçon et de Sainte-Croix notamment).

L'efficacité de ces mesures est éminemment variable en fonction de la réalité du contrôle effectué et surtout de la prise en charge qui en est faite par les personnes touchées elles-mêmes. Mais il est certain qu'elle a été déterminante 


\begin{tabular}{|c|c|c|c|c|}
\hline \multirow{2}{*}{ Bassins } & \multicolumn{2}{|c|}{ Volumes $\left(\mathrm{Mm}^{3}\right)$} & \multirow{2}{*}{ Financement } & \multirow{2}{*}{ Coût (MF) } \\
\hline & Prévus & lâchés & & \\
\hline Garonne-Tarn & 40 & 26,75 & Fonds de garantie des calamités agricoles & 11,8 \\
\hline Aude & 2,2 & 0,45 & Fonds de garantie des calamités agricoles & 0,11 \\
\hline Durance & 100 & 95 & Fonds de garantie des calamités agricoles (dont & \\
\hline & & & $\begin{array}{l}40 \mathrm{Mm}^{3} \text { gratuits compte tenu des économies } \\
\text { réalisées au printemps par les agriculteurs) }\end{array}$ & \\
\hline Lot & 30 & 27,7 & Entente interdépartementale & non chiffré \\
\hline Vézère & 6 & 6,4 & Industriels (Papeterie de Condats) & 0,574 \\
\hline Vienne & 6 & 1,13 & Industriels (Aussedat-Rey et SGPL) & 0,124 \\
\hline Aulne & 1 & 1 & Gratuit (AEP) & \\
\hline Total & 185,2 & $\begin{array}{l}89,73 \\
68,7\end{array}$ & $\begin{array}{l}\text { Indemnisés } \\
\text { Gratuits ou non chiffrés }\end{array}$ & 16,208 \\
\hline
\end{tabular}

pour permettre, par exemple, la prolongation de certaines irrigations des quelques jours ou semaines indispensables pour sauvegarder l'essentiel d'une récolte sans réduire la production de façon trop sensible.

\section{b) Les mesures prises pour accroitre la ressource}

Les réserves constituées par E.D.F. ont été sollicitées massivement, soit au titre des accords permanents conclus avec cet établissement public, soit au titre de mesures exceptionnelles négociées pour l'année 1989.

- les accords permanents : plusieurs bassins bénéficient de façon permanente de fournitures d'eau à partir des

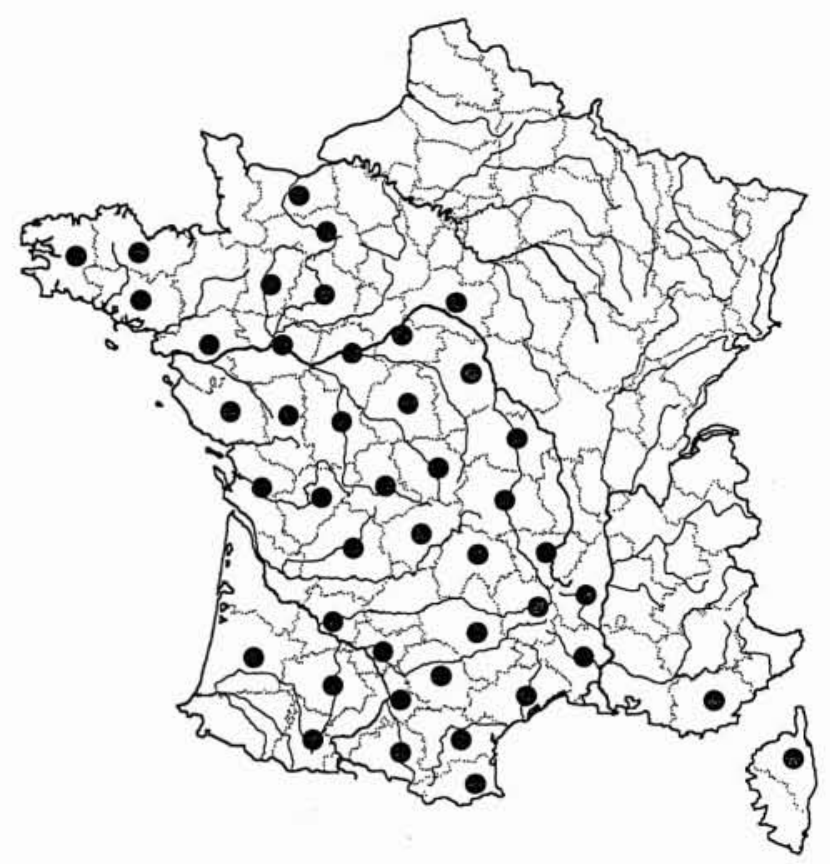

departements ayant pris, sur tout ou
partie de leur teritoire, des mesures d'économies ou de restriction d'eau

9. Cartes des mesures d'économie d'eau ou de restriction prises au cours de l'été 1989. retenues exploitées par E.D.F., dans le cadre de conventions signées principalement avec le ministère de l'agriculture et de la forêt pour les besoins de l'irrigation mais également avec d'autres utilisateurs ;

— des mesures exceptionnelles ont été négociées en 1989 et ont permis la mise à disposition de volumes supplémentaires. Les lâchures ont été effectuées par E.D.F., soit à titre grâcieux, pour l'alimentation en eau potable, soit en contrepartie d'indemnisations, pour l'irrigation ou les besoins industriels.

Ces différentes mesures, conclues à la suite de négociations menées au niveau local, sont rappelées dans le tableau ci-dessus.

On notera que l'indemnisation versée à E.D.F. en contrepartie des mesures exceptionnelles qui allaient audelà des obligations prévues dans les cahiers des charges, a été calculée en tenant compte du coefficient énergétique de chaque ouvrage et de la différence de valorisation $\mathrm{du}^{3}$ d'eau lâché en été par rapport au $\mathrm{m}^{3}$ utilisé au mois de décembre.

\section{Les conséquences sur le milieu naturel}

\subsection{La qualité des eaux superficielles}

Le milieu naturel a particulièrement souffert de la sécheresse de 1989.

La faiblesse des débits (qui accroît la concentration de la pollution chronique), la forte chaleur (qui a tendance a diminuer le taux d'oxygène dissous) et la durée d'ensoleillement (qui favorise la prolifération algale) ont entraîné des niveaux exceptionnels de pollution. Au début du mois d'août est apparue une eutrophisation de très grande ampleur dans les rivières et lacs de Franche-Comté, Limousin et surtout Adour-Garonne et Bretagne.

L'analyse détaillée de l'évolution de la qualité des eaux superficielles est à faire et demandera du temps. Une étude sommaire menée par l'agence de bassin "AdourGaronne » présente cependant des résultats provisoires intéressants : 
- il semble que la sécheresse dans le sud-ouest ait accentué l'eutrophisation dans les rivières où elle se produit habituellement et qu'elle l'ait étendue à des cours d'eau épargnés jusqu'à un passé récent ;

- en ce qui concerne la pollution physico-chimique classique (température, $\mathrm{O}^{2}$ dessous, $\mathrm{DBO} 5$, ammoniaque, phosphates) il semblerait que les impacts des sécheresses de 1976 et de 1989 soient comparables. Ce résultat qui peut paraître surprenant a priori s'explique vraisemblablement par les efforts de dépollution réalisés depuis 1976.

En revanche, on observe une nette dégradation de la qualité (surtout pour l'ammoniaque et les phosphates) si l'on compare 1989 à une année normale du point de vue de la pluviométrie, à savoir 1988 .

Cette analyse provisoire, limitée au bassin AdourGaronne, semble bien refléter la réalité observée sur de nombreux cours d'eau des autres bassins : Seine à l'aval de Paris, Vienne, cours d'eau bretons et normands.

La dégradation de la qualité est également accentuée lors des épisodes orageux qui suivent une longue période sans pluie. Le lessivage des sols apporte alors brutalement au milieu une charge polluante très importante qui se traduit souvent par de fortes mortalités piscicoles.

Il faut heureusement noter que le nombre de pollutions accidentelles a été relativement limité alors que la reprise industrielle du mois de septembre et la campagne de vendanges suscitaient beaucoup de craintes. Il faut probablement voir là l'effet combiné de la plus grande vigilance des services exerçant la surveillance et le sens des responsabilités de la plus grande partie des collectivités locales, des industriels et des viticulteurs qui, constatant la situation, ont renforcé le contrôle des installations, ou différé certaines opérations d'entretien ou certaines productions.

\subsection{Le bilan du point de vue piscicole}

La température, la faiblesse des concentrations en oxygène dissous ont rendu la vie piscicole particulièrement difficile au cours de l'été.

L'assèchement de plusieurs milliers de $\mathrm{km}$ de cours d'eau salmonicoles situés en tête de bassin n'a fait qu'aggraver la situation.

Celle-ci était déjà perturbée dès la fin de l'hiver. En février, il était déjà noté que la faiblesse des débits favorisait le gel des rivières, entraînant une diminution des surfaces des frayères, ce qui a entravé la reproduction des salmonidés et des brochets.

La remontée des migrateurs a été fortement limitée voire rendue impossible au droit de certains ouvrages, la quantité d'eau des installations de franchissement étant insuffisante : à la mi-août, sur l'Elorn (Bretagne) on dénombrait la remontée de 400 saumons seulement, au lieu du millier habituel.

Malgré de nombreuses pêches de sauvetage, la mortalité piscicole est estimée à plusieurs centaines de tonnes. Les éco-systèmes aquatiques risquent d'être perturbés pendant longtemps.

Les régions les plus concernées sont évidemment celles qui connaissent le déficit hydrique le plus important.
A titre d'exemple on notera :

- 30 tonnes de poissons morts sur le Sor, affluent du Tarn ;

- 10 tonnes sur la Vézère ;

- plusieurs tonnes d'anguilles mortes en Bretagne: Aulne, Doufine, Aven.

\section{Les conséquences pour l'alimentation en eau pota- ble}

Les consommateurs d'eau ont finalement été peu touchés par la sécheresse. La plupart des problèmes rencontrés ont été limités à certaines régions où les difficultés sont de toute manière chroniques : hauts villages de Corse, Corbières, Cévennes. Dans ces régions, chaque été un peu sec amène son lot de difficultés : l'été 1989 a simplement été un peu plus intense que la normale. Il faut noter que beaucoup de difficultés constatées procèdent plus de dysfonctionnements techniques ou de sous-dimensionnement des ouvrages (captages de sources sans effet de rabattement, crépines de pompes trop hautes, mauvais état des installations...) que d'une indisponibilité réelle de la ressource.

On signalera le cas des îles du Morbihan qui ont dû êtré alimentées par bateau, dès le mois de juin.

Ce constat positif est essentiellement le résultat des travaux réalisés depuis la précédente sécheresse de 1976. Tirant les enseignements de cet épisode, les collectivités ont en effet consenti de gros efforts d'investissements en matière d'interconnexions, de nouvelles sources de production, d'amélioration des filières de traitement. Dans le même temps, des barrages-réservoirs ont été construits ; ils ont largement permis de soutenir des débits d'étiages qui, sans eux, auraient été particulièrement bas (Seine, Loire, Bretagne, Vendée).

Il faut aussi considérer que des conséquences graves sur l'alimentation des populations ont été évitées grâce à l'intense mobilisation des services de production et de distribution d'eau qui ont su mettre en œuvre, lorsqu'il le fallait, les solutions de dépannage indispensables.

Cependant, il ne faut pas se limiter à ce constat relativement positif, en effet :

- les difficultés de traitement dues à la forte pollution organique des eaux brutes ont été considérables, notamment dans le sud-ouest. Si les véritables incidents ont été limités (banlieue de Montluçon, Villeneuve-sur-Lot), beaucoup d'autres usines ont fonctionné à la limite du possible ou du raisonnable;

- les fortes chaleurs ayant entraîné de très fortes consommations, les installations ont souvent fonctionné au maximum de leurs possibilités. La chance a voulu qu'aucun incident technique ne survienne à ce moment, mais il faut noter la grande fragilité d'un système sollicité près de $24 \mathrm{~h} / 24$;

- une région entière comme la Bretagne n'a évité des difficultés majeures qu'en raison du retour in extremis des pluies à la fin du mois d'octobre. Quinze jours de sécheresse supplémentaires eussent probablement entraîné des ruptures d'alimentation, notamment dans le sud Finistère. 
Pour toutes ces raisons, il faut se garder d'afficher un trop grand optimisme même si l'on peut légitimement se réjouir des résultats des efforts consentis dans le passé.

\section{Les conséquences pour l'agriculture}

L'agriculture a été l'une des principales victimes de la sécheresse même si celle-ci a permis de bonnes productions de fruits ou de vins de qualité.

Le bilan complet ne peut encore être fait. Mais l'on sait d'ores et déjà que la sécheresse a eu des effets négatifs sur l'élevage (moins de fourrage), les récoltes de pommes de terre, de maïs, de betteraves et d'oléagineux.

Bien entendu les cultures non irriguées ont été les plus touchées.

Face a cette situation, le gouvernement a mis en place un train de mesures exceptionnelles en faveur des agriculteurs victimes de la sécheresse. Ce dispositif vise à compléter les mesures prises dans le cadre des procédures ordinaires (prêts calamités bonifiés distribués par le crédit agricole, indemnisation par le fonds national des calamités agricoles).

Au début de mois de décembre, 69 départements étaient reconnus comme sinistrés.

Au total une somme de $1800 \mathrm{MF}$ a été affectée à ce dispositif.

\section{Les conséquences pour la production électrique}

Le taux de remplissage des réservoirs hydroélectriques s'est maintenu tout au long de l'été à un niveau très inférieur à ce qu'il est en année moyenne. Au 9 novembre, il était de $56 \%$ alors qu'il est en moyenne de $75 \%$ à la même époque.

Ce déficit s'est traduit sur le plan énergétique par une indisponibilité de puissance de 2000 à $3000 \mathrm{MW}$ pour les centrales hydroélectriques.

Le déficit hydraulique a également affecté le parc de production thermique nucléaire puisqu'en moyenne $700 \mathrm{MW}$ sont restés indisponibles par suite de débits insuffisants dans les fleuves et de respect des températures de rejet entre le 15 juin et le 15 octobre.

Les pertes de production imputables à la sécheresse ont été estimées au 30 septembre à environ $10 \mathrm{TWh}$ (15 TWh environ sur l'année). Cette perte a été compensée par de lénergie produite par des centrales thermiques nucléaires et classiques ainsi que, pour une faible proportion, par des achats à l'étranger.

Toujours en ce qui concerne la production hydroélectrique, celle des producteurs autonomes a été réduite de moitié par rapport à une année normale dans le sud et l'ouest du pays, ce qui représente une diminution qui est estimée a 1 ou $2 \mathrm{TWh}$. De nombreux producteurs se retrouvent ainsi en situation financière délicate.

\section{Les conséquences pour le réseau de voies navigables}

Les principales gênes à la navigation fluviale ont été rencontrées sur les canaux à bief de partage.

En effet, le niveau d'eau de ces biefs est la plupart du temps assuré par des barrages-réservoirs, alimentés par les précipitations.

Dès le début de l'année, les réserves dans certaines retenues étaient inférieures à la moyenne (en particulier en Bourgogne ou dans le Midi-Pyrénées), aussi le manque d'eau s'est fait cruellement sentir au cours de l'été.

Le premier canal atteint fut le canal du Midi, où, dès le 20 février 1989, le mouillage n’a plus été garanti entre Toulouse et Carcassonne.

Par la suite, la situation ne s'améliorant pas, la navigation a été interrompue sur le bief de partage le 17 avril 1989, en prévision d'un été sec.

D'autres arrêts de la navigation, pour des raisons identiques, sont intervenus au cours de l'été et au début de l'automne sur les canaux suivants (fig. 10) :

- canal de Briare et latéral à la Loire ;

- canal de Bourgogne;

- canal de Nivernais ;

- canal de la Marne à la Saône.

Les canaux les plus touchés par la sécheresse sont les canaux de type Freycinet (petit gabarit) et de gabarits inférieurs (canal de Nantes à Brest).

Mais le débit de certaines rivières ayant fortement baissé, de nombreux incidents sont apparus sur les sections navigables de ces dernières ou les canaux alimentés par ces rivières :

- canal de la Robine alimenté par l'Aude, fermé pendant 2 mois ;

- canaux de l'Est et de la Marne au Rhin.

Les voies navigables à grand gabarit (Seine, Rhin, Rhône et voies navigables du Nord) n'ont pas eu à souffrir des effets de la sécheresse.

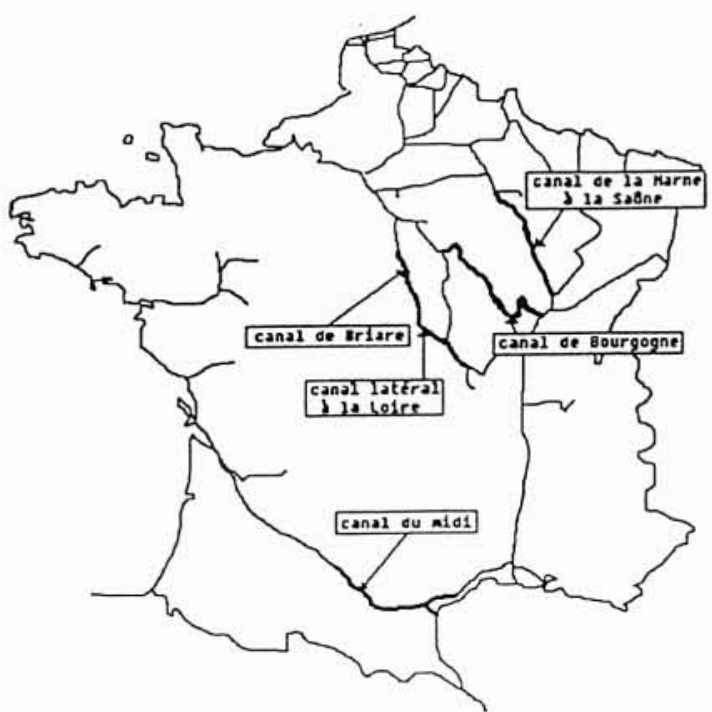

10. Voies navigables les plus touchées par la sécheresse (Source Ministère des transports). 


\section{Les conséquences pour le tourisme}

Les conséquences économiques du manque d'eau sont difficiles à chiffrer. La navigation de plaisance a été très gênée par les interruptions de trafic mais celui-ci s'est reporté en partie sur des sections de canaux ouvertes.

Le manque de neige a été très pénalisant pour les stations de montagne pendant le début de l'hiver 19881989 , où les professions saisonnières ont beaucoup souffert.

\section{Les conséquences pour les feux de forêt}

La France comporte environ 15 millions d'hectares de forêt. Chaque année 45000 ha en moyenne sont la proie des flammes. Selon les années, la forêt méditerranéenne (zone Prométhée) représente en moyenne entre $70 \%$ et $80 \%$ de cette valeur.

Parmi les conditions qui favorisent l'apparition des feux de forêts, la sécheresse n'est pas le seul facteur explicatif (le vent, notamment, joue un rôle important) mais elle est l'un des plus importants.

1976 avait été une année sèche pour le nord de la France. Les feux de forêts avaient gagné les départements généralement épargnés, mais moins aptes à faire face. En 1989, le sud aura été particulièrement touché :

56000 ha pour la zone Prométhée pour un total de 77000 ha (chiffres au 20 novembre 1989).

On observera cependant que la surface parcourue par le feu en 1949 (année de sécheresse comparable) avait été considérablement supérieure : 300000 ha.

L'amélioration des moyens de prévention, de surveillance et de lutte a donc en partie porté ses fruits.

\section{Enseignements à tirer de la sécheresse de 1989}

\subsection{Sur le plan réglementaire et institutionnel}

- La sécheresse a mis en lumière de façon éclatante les carences de la réglementation: impossibilité de quantifier les prélèvements dans les eaux non domaniales, pas de maîtrise des prélèvements en nappe souterraine. L'absence d'outils réglementaires autorisant la puissance publique à répartir les faibles ressources au mieux de l'intérêt général s'est fait cruellement sentir.

Ce constat, déjà posé dans plusieurs rapports parlementaires (M. Malandain; M. Tenaillon) rend encore plus opportun le projet de loi sur l'eau en cours d'étude.

- En période de crise les interdictions ou limitations de certains usages ont un caractère global : interdiction sur tout un département, tout le long d'une rivière. Une connaissance plus fine de la ressource et la prise en compte précise de certaines conditions hydrauliques concernant les eaux souterraines (inertie et déphasage entre eaux superficielles et eaux souterraines) devraient permettre une plus grande modulation des mesures administratives d'interdiction de l'irrigation et ainsi limiter les conséquences économiques de la sécheresse.

- Le gouvernement doit présenter au Parlement un bilan de l'application de l'article 410 du code rural (débits réservés; loi pêche de 1984). L'analyse de la situation hydrologique constatée en 1989 fournira des éléments d'information intéressants pour faire ce bilan et apprécier la capacité des débits réservés légaux à assurer le respect des objectifs de qualité et la protection des milieux aquatiques et des besoins piscicoles.

\subsection{La demande en eau}

Si l'équilibre besoins-ressources a été rompu pendant une très longue période, la responsabilité en revient certes à une pluviométrie anormalement basse, mais également à une évolution de la demande en eau qui, depuis 10 ans, a été plus rapide que celle des ressources.

Cette forte croissance de la demande est, en quasitotalité, due aux besoins de l'irrigation.

Dans la région Midi-Pyrénées la surface irriguée a été multipliée par 1,7 de 1979 à 1988. En Aquitaine, ce coefficient multiplicateur est de 1,8. Dans le département de la Vienne, il est proche de 3,7 .

\begin{tabular}{|c|c|c|c|}
\hline & Midi-Pyrénées & Aquitaine & Vienne \\
\hline 1970 & 60473 ha & 67663 ha & \\
\hline 1979 & 123081 ha & 128050 ha & 4994 ha \\
\hline 1988 & 209891 ha & 229658 ha & 18326 ha \\
\hline
\end{tabular}

Face à une telle progression, on peut craindre que certains programmes de développements de la ressource engagés ici ou là, l'aient été sur des hypothèses d'ores et déjà dépassées.

Pourtant la poursuite de cet effort est indispensable si l'on veut concilier la survie et le développement de l'économie agricole avec le respect et l'amélioration de la qualité du milieu "eau». Mais il convient également de mieux apprécier que dans le passé les conditions générales d'évolution des besoins en eau d'irrigation, d'en chiffrer le degré de rentabilité économique tant au niveau de chaque exploitation qu'au niveau global (internalisation du coût d'aménagement de la ressource), et de définir, à la lumière de ces analyses, le degré de défaillance vis-à-vis duquel il est raisonnable de se prémunir.

C'est une véritable expertise de la politique menée jusqu'à présent qui doit être faite avec toutes les parties intéressées, pour garantir la pertinence globale des choix arrêtés mais également leur acceptabilité par les populations concernées : la période de sécheresse que nous venons de vivre peut en effet réactiver les conflits latents entre aménageurs de la ressource et protecteurs de la nature. 


\subsection{Les économies d'eau}

\section{a) Economie d'eau domestique}

On estime qu'en moyenne $40 \%$ de l'eau produite sont perdues sous forme de fuites, pour moitié sur le réseau et pour moitié chez l'usager. Cette moyenne marque de grandes plages de variation:

— si les réseaux urbains neufs peuvent atteindre des rendements supérieurs à $90 \%$, le rendement des réseaux anciens peut être inférieur à $50 \%$;

- chez l'usager, un bon entretien peut annuler toute perte d'eau, en revanche une fuite sur une chasse d'eau peut représenter $150 \mathrm{~m}^{3} / \mathrm{an}$.

En volume annuel, et pour toute la France, ces fuites représenteraient donc un volume de près de $1200 \mathrm{Mm}^{3}$. Il en résulte de multiples conséquences :

- frais de fonctionnement dépensés inutilement ;

- nécessité de surdimensionner les installations ou difficultés en période de consommation de pointe (cela a été le cas au cours de l'été 1989);

- arrivée dans les stations d'épuration d'eaux excédentaires trop diluées, ce qui rend le traitement moins performant ;

— prélèvements inutiles dans la ressource en eau : dans le cas de collectivités prélevant au fil de l'eau, les fuites ne peuvent être assimilées (en totalité) à une perte dans la mesure où les usagers de l'aval peuvent la réutiliser. Elles impliquent cependant la mise à disposition du principal préleveur d'un volume d'eau plus important que nécessaire. En revanche, dans deux cas fréquents, ces fuites peuvent s'interpréter comme une perte sur la ressource en eau, particulièrement préjudiciable en période de sécheresse :

- les secteurs où les prélèvements s'effectuent en eau souterraine : ceux-ci représentent $60 \%$ des prélèvements des collectivités locales. Dans ce cas, les pertes chez l'usager gagnent la plupart du temps le réseau d'eau superficielle situé plus à l'aval et sont donc effectivement «perdues " pour la ressource disponible. C'est le cas, par exemple, des nappes du Languedoc-Roussillon dont le niveau baissait de 1 mètre par semaine au mois d'août 1989. Au total pour toute la France, les volumes ainsi prélevés inutilement seraient de $700 \mathrm{Mm}^{3} /$ an. Il en est de même pour les pertes sur le réseau qui, bien que regagnant le milieu souterrain, ne le font qu'en un lieu généralement différent du lieu de prélèvement.

- les secteurs alimentés par une ou plusieurs réserves d'eau brute qui ne sont pas, la plupart du temps, le réceptacle des eaux usées de la collectivité. Dans ce cas, les fuites s'interprètent bien comme une consommation inutile de la ressource qui s'épuise plus vite que ce qui serait strictement nécessaire.

Pendant la sécheresse de 1989, ce cas de figure s'est rencontré sur le Cher (alimentation de Montluçon et barrage de Rochebut), dans la vallée de l'Aulne (réserve de Brennilis), à Rennes, Limoges, etc...

En dehors de toute considération économique, un effort de lutte en faveur des économies d'eau devrait avoir un impact positif vis-à-vis de la ressource en eau, compte tenu de l'ampleur des volumes mis en jeu.

\section{b) Economie d'eau agricole}

La consommation nette de l'agriculture est très importante en période d'étiage (dans le sud de la France et pendant l'été, l'agriculture représente plus des deux tiers des consommations nettes). Toute économie réalisée dans ce domaine se traduira immédiatement par un accroissement des ressources disponibles soit pour d'autres usages, soit pour l'irrigation elle-même.

Des économies sont possibles et ont déjà été réalisées. Dans les zones des régions méditerranéennes, où sont intervenus à la fois une modernisation des infrastructures d'adduction et de desserte et un abandon de l'irrigation gravitaire au profit de l'irrigation sous-pression, on peut estimer que le prélèvement sur la ressource en eau est trois à quatre fois moindre (une telle reconversion n'est cependant pas souhaitée ni possible partout).

Il importe donc de poursuivre les efforts :

- par la réhabilitation et la modernisation des infrastructures existantes ;

- par la recherche de techniques plus économes de distribution de l'eau en tête des parcelles et sur leur surface;

- par la recherche agronomique afin de mieux connaître les ressources du sol ainsi que la physiologie et les besoins des cultures selon leurs différentes phases de développement ;

- par la formation des agriculteurs à la conduite d'une irrigation la plus efficace possible et à la gestion d'un assolement sous contrainte de débit et de volume d'eau maximum ;

- par la gestion automatique des réservoirs et des infrastructures de desserte en temps réel, dans les régions d'irrigation collective: l'expérience acquise et les réalisations effectuées par la Société du Canal de Provence ou la Compagnie d'Aménagement des Coteaux de Gascogne montrent qu'une anticipation des besoins d'irrigation, jointe à une meilleure utilisation des débits naturels disponibles, permet d'économiser de l'ordre de $20 \%$ des volumes stockés.

\subsection{La connaissance et le développement des ressources en eau}

\section{a) La connaissance des ressources en eau}

La période de crise induite par la sécheresse de 1989 a mis en lumière le besoin de connaissance en temps réel du niveau de la ressource. Cette connaissance n'a pu être acquise que grâce à la mise en œuvre des procédures exceptionnelles qui ne peuvent être pérennes. Il y a donc lieu de poursuivre et d'accentuer le développement et la modernisation des réseaux piézométriques et hydrométriques pour atteindre cet objectif. Simultanément devra être conduit un effort de prévisions des étiages à partir de modèles scientifiques en cours d'élaboration. 


\section{b) Le développement des ressources en eau}

Quels que soient les efforts qui seront faits pour mieux maîtriser la croissance de la demande et pour lutter contre le gaspillage, une augmentation des volumes disponibles est indispensable, au moins pour que le milieu naturel ne subisse plus aussi durement qu'en 1989 les conséquences des sécheresses futures.

L'étiage de 1989 a montré sur les bassins de la Seine et de la Loire le rôle positif des barrages de soutien d'étiage. A contrario, on a pu constater sur le bassin AdourGaronne les graves défaillances dues à un manque de réserves, justifiant encore d'avantage le programme décennal de développement des ressources en eau (PDRE).

Le développement des ressources, à condition d'être établi avec soin et dans le respect des équilibres fondamentaux, peut être un facteur déterminant de la qualité du milieu.

Pour développer ces ressources, il sera intéressant d'examiner avec E.D.F. l'extension de montages financiers déjà opérationnels qui permettent de bénéficier de ressources supplémentaires soit par la participation au financement d'ouvrages à construire, soit par l'achat d'une tranche d'eau sur des ouvrages existants.

Pour définir ou actualiser ces programmes de développement des ressources, il conviendra d'analyser très précisément la situation réelle de l'été 1989, secteurs par secteurs.

Cet effort d'équipement doit cependant être complété par une réflexion en profondeur à propos de l'impact sur les ressources en eau d'un certain nombre de décisions d'aménagement du territoire: conséquences du gel des terres, de la politique forestière, des politiques anciennes de remembrement, etc...

\subsection{La sécurité de l'alimentation en eau potable}

On a vu que l'alimentation en eau des populations avait, somme toute, été assurée de façon globalement satisfaisante. Mais on a également constaté qu'il fallait se garder de tout optimisme exagéré dans la mesure où le système a été dans de nombreuses régions et pour de longues semaines, au bord de la rupture. Le sous-dimensionnement ou la vétusté, et par voie de conséquence la fragilité, de certains réseaux, ont été ainsi souvent mis en évidence, notamment en zone rurale.

Par ailleurs, les services administratifs se sont trouvés quelque peu démunis pour anticiper l'évolution de la situation en cas de prolongation de la période de sécheresse : connaissance approximative des zones à risque, impossibilité de mesurer précisément le risque réel de défaillance et de définir la période au cours de laquelle cette défaillance pourrait survenir.
Il convient donc de poursuivre les efforts pour améliorer la fiabilité et la sécurité de l'alimentation en eau potable des populations : diversification de la ressource, appel plus important aux eaux souterraines, amélioration des dispositifs de prélèvement et de traitement...

Les programmes de travaux à engager demanderont un effort financier très important de la part des collectivités locales et des autres partenaires financiers. Il ne faut pas sous-estimer les difficultés qui seront rencontrées dans la mise en œuvre de ces programmes pourtant indispensables : cet effort doit être fait à un moment où les nombreux ouvrages construits avant la seconde guerre mondiale ou dans les années cinquante nécessiteront un renouvellement, ce qui entraînera des besoins de financement considérables. A cet égard la baisse continue des dépenses d'investissement des administrations publiques consacrées à la mobilisation de la ressource au profit des dépenses de fonctionnement est préocupante puisque c'est à un véritable retournement de tendance qu'il faudra assister dans les prochaines années. Il est certain que les dépenses à consentir auront des conséquences en matière de prix de l'eau. Celui-ci reste relativement faible (compte tenu du service rendu) notamment si on le compare au prix d'autres services publics ou simplement au prix de l'eau embouteillée. D'autres pays européens pratiquent des prix de l'eau beaucoup plus élevés qu'en France : les élus français doivent également résoudre ce choix de politiques locale et nationale que la sécheresse remet au premier plan de l'actualité.

\subsection{La lutte contre la pollution}

Les événements de l'été 1989 ont montré l'évidente relation entre les problèmes de quantité et de qualité. Les premières difficultés majeures rencontrées par l'alimentation en eau potable ont d'ailleurs été dues à la mauvaise qualité de la ressource.

Il est donc absolument indispensable de poursuivre l'effort entrepris depuis de nombreuses années dans le domaine de la dépollution, de l'étendre à de nouvelles pollutions non prises en compte suffisamment jusqu'à présent (nitrates, phosphates, pollutions apportées par le ruissellement des eaux pluviales), et de le rendre pleinement efficace par une meilleure gestion des installations existantes (réseaux d'eaux usées, stations d'épuration).

Les moyens financiers à mobiliser seront très importants dans la mesure où, par exemple, le montant des travaux à réaliser pour le traitement des eaux pluviales est estimé, en première analyse, à une valeur du même ordre de grandeur que le montant consacré au traitement des pollutions classiques. 


\section{Conclusions}

La sécheresse de 1989, dont le début remonte en fait à la fin 1988 et qui se poursuit encore début 1990 au moment où ces lignes sont écrites, marquera probablement fortement les politiques de l'eau des années 1990.

Si l'alimentation en eau potable, l'usage de l'eau le plus indispensable, a été finalement assurée sans déboires de grande envergure - c'est d'ailleurs à mettre au crédit des décisions prises depuis les précédentes sécheresses - il faut reconnaître que les limites du système ont été atteintes dans plusieurs régions de France. Des progrès restent donc à faire pour garantir à nos réseaux de production et de distribution d'eau une fiabilité et une sécurité renforcées.

Par ailleurs, certains secteurs économiques ont souffert : l'agriculture, la production électrique, la navigation de plaisance, etc...: les pertes se chiffrent en milliards de francs.

Enfin, le milieu naturel a particulièrement été touché, qu'il soit aquatique ou terrestre, puisque l'année 1989 est également une année exceptionnelle pour les feux de forêt.

Un tel événement rappelle brutalement qu'une ressource naturelle que l'on pourrait croire inépuisable sous nos climats tempérés reste soumise à des variations d'une amplitude telle qu'elle interroge le fonctionnement même de la société et les objectifs qu'elle poursuit.

Il convient donc de mener l'analyse des programmes de travaux qu'il faudra engager pour mieux garantir la sécurité de l'approvisionnement en eau et mieux protéger le milieu naturel. Mais cette analyse serait insuffisante si elle n'était pas complétée par une réflexion plus fondamentale sur les attentes de notre société vis-à-vis de sa ressource en eau, sur le degré de défaillance acceptable et, par voie de conséquence, sur le degré d'artificialisation du cycle de l'eau qui en découle.

Une véritable "remise à plat " de la politique de l'eau menée par les différents partenaires apparaît donc nécessaire. C'est précisément pour cette raison qu'on été décidées les Assises de l'Eau qui, pendant deux ans, permettront à chacun des acteurs de participer à ce débat. 


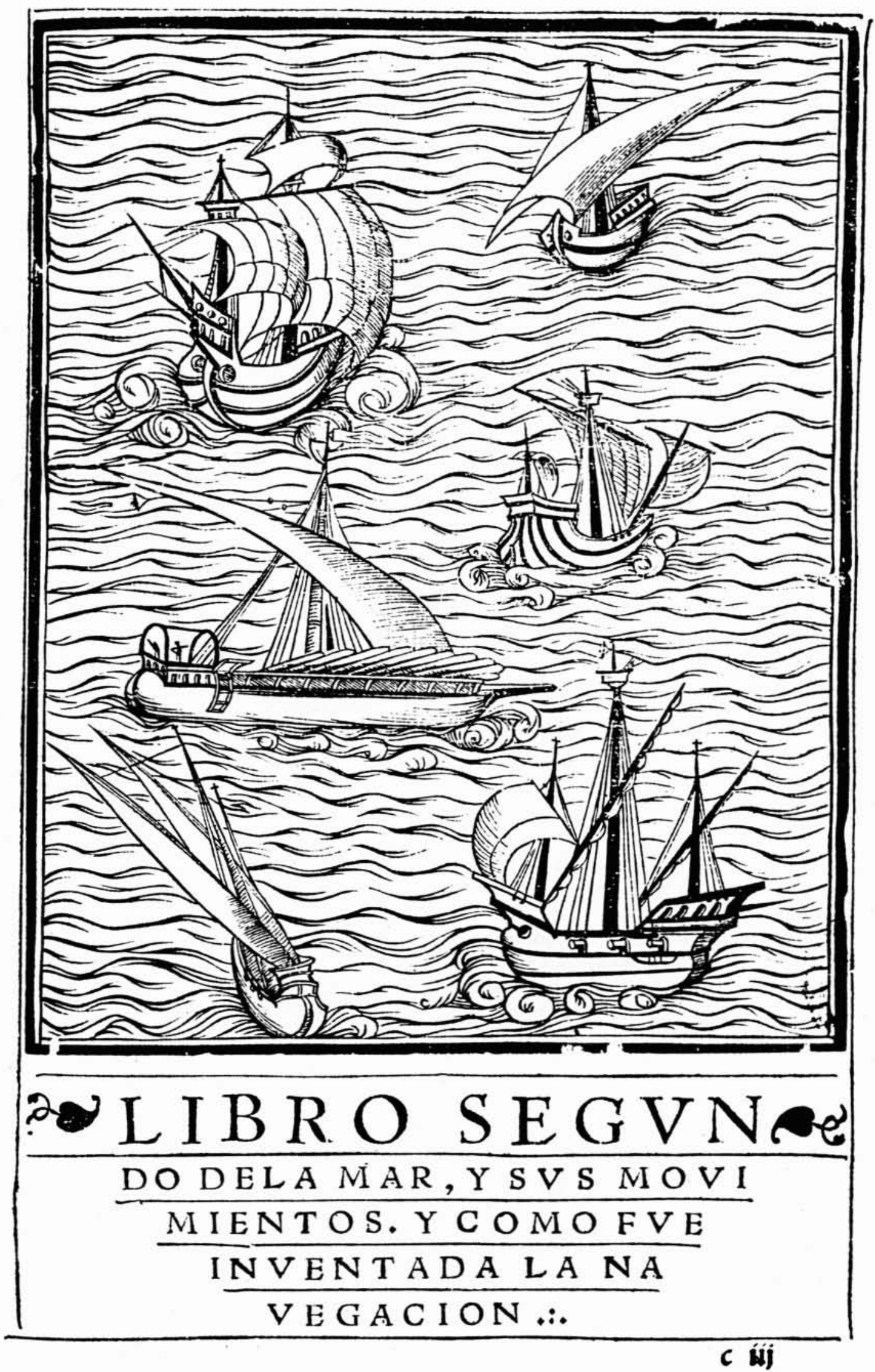

